\title{
Architecture of a Web of Things eHealth framework for the support of users with chronic diseases
}

\author{
Carlos A. Velasco ${ }^{1}$ \\ Fraunhofer Institute for \\ Applied Information \\ Technology (FIT), St. Augustin, \\ Germany \\ carlos.velasco@fit.fraunhofer.de
}

\author{
Yehya Mohamad \\ Fraunhofer Institute for \\ Applied Information \\ Technology (FIT), St. Augustin, \\ Germany \\ yehya.mohamad@fit.fraunhofer.de
}

\author{
Philip Ackermann \\ Fraunhofer Institute for \\ Applied Information \\ Technology (FIT), St. Augustin, \\ Germany \\ philip.ackermann@fit.fraunhofer.de
}

\begin{abstract}
This paper presents an architecture, system components, applications and interfaces of a Web of Things framework for supporting home hospitalization of users with chronic diseases. Home hospitalization intends to provide health care at same level and quality at the home of the patient same as it is provided at a hospital.

After an introductory section, the document presents a global overview of the design philosophy, together with the main actors involved. Then the different layers of the system and their components are depicted. Finally, a walkthrough on the different technologies that will be used for the implementation of an instance of the described architecture is being provided.

This paper builds upon known requirements for home hospitalization and serves as basis for the implementation of instances of such system.
\end{abstract}

\section{Keywords}

Internet of Things (IoT); Web of Things (WoT); health care; sensor; hospital; smart home; home hospitalization.

\section{INTRODUCTION}

The internet of things (IoT) is the internetworking of physical devices, vehicles, buildings and other items embedded with electronics, software, sensors, actuators, and network connectivity that enable these objects to collect and exchange data ${ }^{1}$. The IoT allows objects to be sensed and/or controlled remotely across existing network infrastructures ${ }^{2}$, creating opportunities for more direct integration of the physical world into computer-based

Permission to make digital or hard copies of all or part of this work for personal or classroom use is granted without fee provided that copies are not made or distributed for profit or commercial advantage and that copies bear this notice and the full citation on the first page. Copyrights for components of this work owned by others than the author(s) must be honored. Abstracting with credit is permitted. To copy otherwise, or republish, to post on servers or to redistribute to lists, requires prior specific permission and/or a fee. Request permissions from Permissions@acm.org.DSAI 2016, December 01 - 03, 2016, Vila Real, Portugal

Copyright is held by the owner/author(s). Publication rights licensed to ACM. ISBN 978-1-4503-4748-8/16/12 \$15.00

DOI: http://dx.doi.org/10.1145/3019943.3019951

\footnotetext{
${ }^{1}$ http://www.itu.int/en/ITU-T/gsi/iot/Pages/default.aspx 2

https://hbr.org/resources/pdfs/comm/verizon/18980_HBR_Veriz on_IoT_Nov_14.pdf
}

systems, and resulting in improved efficiency, accuracy and economic benefit. IoT devices can be used to enable remote health monitoring and emergency notification systems. These health monitoring devices can range from blood pressure and heart rate monitors to advanced devices capable of monitoring specialized implants, such as pacemakers, electronic wristbands or advanced hearing aids [1]. Specialized sensors can also be equipped within living spaces to monitor the health and general well-being of elderly with chronic diseases, while also ensuring that proper treatment is being administered and assist people regain lost mobility via therapy as well. More and more end-to-end health monitoring IoT platforms are coming up for chronic patients, helping in the care planning and managing of health vitals and recurring medication requirements ${ }^{3}$. Such an IoT system is a complex distributed system involving different development platforms and teams and requires a reference architecture as a guide. The identification of a reference architecture can lead to a faster, more focused development and an exponential increase of IoT-related solutions. These solutions can provide a strategic advantage to mature economies, as new business models can leverage those technological solutions providing room for economic development ${ }^{4}$.

\section{This paper especially focuses on:}

- The system characteristics

- The system boundaries

- Assumptions and restrictions that are important for the design and implementation

- The high-level design of the system in terms of its main components and how they should interact

This paper is a guide for further detailed design activities and implementation of such instances. It is helpful for architects to use this part of the paper to collaborate with other stakeholders in developing the architecture and to understand the motivation behind architectural decisions so that those decisions can be robustly implemented.

For example, the architect may put constraints on security issues regarding the (type of) data stored in the system. This may appear to be a burden, but it can be justified by the fact that users do not want to put data outside their toolset and only want to use

\footnotetext{
${ }^{3} \mathrm{http}: / /$ www.polycare-project.com/

${ }^{4} \mathrm{http}$ //www.iot-a.eu/public/public-documents/copy_of_d1.2
} 
the system as a local stand-alone tool or plug-in. The rest of the design must adapt to this restriction.

This paper informs as well on how the instances are partitioned or organized, so that the developer team can adapt to the needs of the system. It also gives a high-level outline of such instances and their technical motivations to whoever must maintain and modify the architecture later.

This paper is organized as follows:

- Overview of the architecture: this part outlines the main architectural decisions including a vision of the overall approach, a general overview of instances.

- Architecture Description: this part provides the details of the architecture, describes the different components of the system and their interactions.

- Technologies: this part describes technologies, software libraries and frameworks that will be used for developing of an example instance.

The system architecture shall be designed to be modular and distributed, to facilitate information sharing among networked sites. In addition, emphasis is put on flexibility issues, so that different development process approaches, sensor networks, data storage and technological maturity levels are supported. A modelcentred, component-based, event-driven approach is adopted to ensure architecture portability, fault tolerance, interoperability, and independence between the different building blocks.

We describe an instance as an infrastructure on which home hospitalization tools run and performs tasks in the domain of eHealth. We understand the system as a collaboratively developed tool chain that supports data gathering, persistence, interpretation and presentation to various stakeholders.

\subsection{System's mission}

The mission of the proposed system and its biggest challenge is to provide functionality that supports home hospitalization artefacts. In addition, the system aims at empowering the caregiving activities as well as providing facilities to integrate the activities of all stakeholders participating at the caregiving process at all stages and minimizing the effort of those.

We can summarize these aspects as follows:

- $\quad$ Providing guidance to stakeholders in the caregiving process (patients, doctors, formal and informal caregiver) on how to comply with standards and regulations. This includes the definition of a structured concept and tools to store knowledge rules about the home hospitalisation processes, their interpretations, and the strategies/decisions to comply with them.

- $\quad$ Providing user interfaces to define rules on how the system should react to detected anomalies in the gathered and interpreted data. For instance, sending an SMS to a specific caregiver.

- Supporting the care decision process of physicians according to data gathered and interpreted by the system. The system contains a decision support system and the physician should meet the final decision after careful elaboration.

- Facilitating integration with state-of-the-art eHealth tools. The system core consists of a set of tools that:

- Connecting interfaces to external systems and repositories like $\mathrm{EHR}$, to provide all stakeholders involved in the caregiving process of a patient with the latest information about her. The existing tools need to provide additional value to the home hospitalisation case. Standards like HL7 will be utilised to communicate between existing tools / repositories and the system;

- Creating components from or based on building blocks from partners, which provide additional value to the development of the home hospitalization;

- Extending and utilising open source frameworks; and

- Creating components from scratch and provide new functionality to the development of home hospitalization artefacts.

The system will also provide tools to support managers of the caregiving process by administering care agendas of their patients, any change in one or many agendas will lead to a recalculation of the schedules allowing them to choose between alternative agendas and finally to confirm the elected one. Again, the system will interface with existing tools, techniques and processes.

\section{ARCHITECTURE DESCRIPTION}

\subsection{Architecture layers}

The software system to be developed is divided into five horizontal layers using a service oriented approach: presentation layer, application layer, service layer, data access layer and persistence layer. Those layers are defined as follows:

- Presentation Layer: contains those components related to the user interface of the system.

- Application Layer: contains those components specific to different applications of the system, i.e., the application logic.

- Service Layer: contains reusable services of the system. Those services are used by the applications defined in the application layer.

- Data Access Layer: contains components for accessing the data.

- Persistence Layer: contains components for persisting the data.

Additionally, there are crosscutting concerns, which apply to all of the layers and that are shown vertically next to the layers: access control, security, logging, internationalization and caching. All of the layers and their subcomponents are described in more detail in the following sections (see Figure 1).

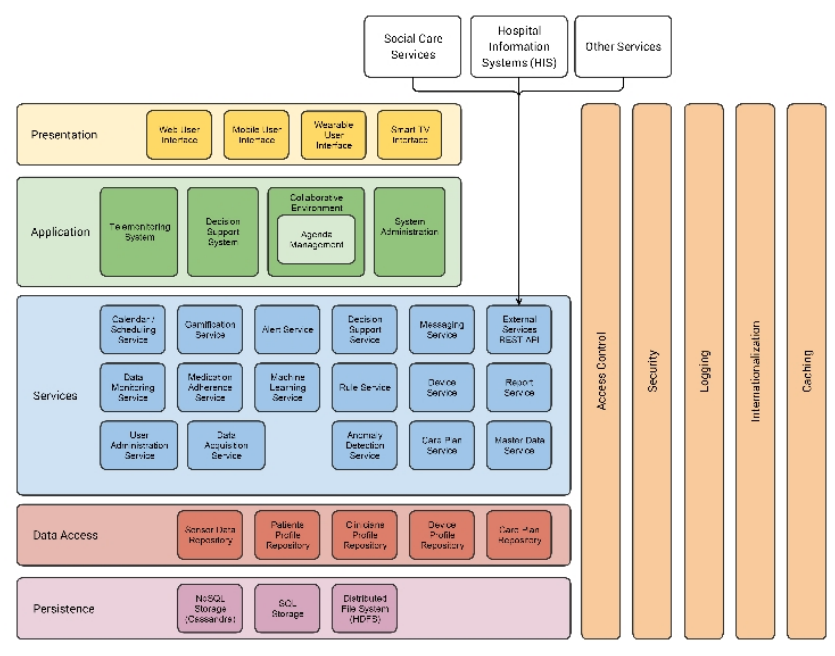

Figure 1: Layered structure of WoT eHealth tool modules. 


\subsubsection{Presentation Layer}

Presentation layer is the top piece of this Architecture (See Figure 1 ), in term of development design and dependencies. It has mainly the scope to create an Interface with the final User of the application. It can take the form of Desktop forms, Web application, mobile application, TV application or any other possible solution provided by technologies. This layer contains the user-oriented functionality responsible for managing user interaction with the system, and generally consists of components that provide a common bridge into the core business logic encapsulated in the application layer ${ }^{5}$. Apart from target technologies there are many general design considerations, recommendations and standards to be considered when talking about the presentation layer e.g. W3C WAI guidelines (WCAG 2.0) tackling accessibility aspects of user interfaces or ISO 9241-11, 110 und -210 tackling the usability of user interfaces etc.

\subsubsection{Application Layer}

The application layer contains concrete application logic.

- Tele monitoring System: application to manage and acquire health data from the patients. It presents different interfaces depending on the different actors accessing the application.

- Decision Support System: application to analyse the health data and create different rules related to care, medication, etc.

- Collaborative Environment: application that will allow all the actors included in the care of the patients to share information and to interact between them.

- System Administration: application for administrating different components of the system, e.g., users, devices.

\subsubsection{Service Layer}

The service layer contains reusable services of the system. It will be designed using a SOA approach (Service Oriented Architecture, see SOA Reference Architecture Technical Standard ${ }^{6}$ ). The list of services presented in Figure 1 is a preliminary approach that will be extended/modified through the development process in the following work packages:

- Alert Service: a service for sending alerts.

- Gamification Service: a service for managing the gamification interfaces.

- Calendar / Scheduling Service: a service for managing calendars and schedulers.

- User Administration Service: a service for administrating and managing the users. This service also allows storing all actions performed by users within the applications.

- Decision Support Service: a service for the decision support.

- Messaging Service: a service for different types of messaging. This service will be capable of sending messages through the system, e.g., as an email or by using a message bus.

- External Services REST API: relevant services will be exposed through a public REST API, so that third parties

\footnotetext{
${ }^{5}$ https://msdn.microsoft.com/en-us/library/ee658109.aspx

${ }^{6} \mathrm{http}: / /$ www.opengroup.org/soa/source-

book/soa_refarch/concepts.htm
}

(like hospital information systems) can easily integrate the services into their systems.

- Data Monitoring Service: a service for monitoring data.

- Medication Adherence Service: a service that supports the adherence of medication.

- Anomaly Detections Service: a service for detecting anomalies.

- Device Service: a service for administrating and managing the devices.

- Rule Service: to process different rules created to manage different events within other services.

- Machine Learning Service: a service that implements or exposes different machine learning algorithms.

- Data Acquisition Service: a service to support the data acquisition for the sensors.

- Care Plan Service: this service allows the management of templates and care services plan. It also allows the configuration of each care plan.

- Master Data Service: it contains operations such as creation, deletion and update of master data used in the application.

- Report Service: it allows management reports used by the application. These are e.g.:

$$
\begin{array}{cl}
\circ & \text { Consent form } \\
\circ & \text { Social assessment } \\
\circ & \text { Discharge report } \\
\circ & \text { User report }
\end{array}
$$

\subsubsection{Data Access Layer}

The data access layer contains components for accessing data. Repositories are an abstraction of the underlying persistence and facilitate access to that layer. The components in this layer can be implemented using Node.js and software libraries that implement JPA concepts. ${ }^{7}$

- Sensor Data Repository: a repository for accessing sensor data.

- Patients Profile Repository: a repository for accessing the profiles of patients.

- Clinicians Profile Repository: a repository for accessing the profiles of clinicians.

- Device Profile Repository: a repository for accessing the profiles of devices.

- Care Plan Repository: a repository that brings together all the care plan management access.

\subsubsection{Persistence Layer}

For persistence, we foresee different types of storages. Some data (e.g., sensor data) will be stored in NoSQL databases (a.k.a. big data storages), while some data (e.g., user profiles) will be stored in SQL databases.

\footnotetext{
${ }^{7}$ https://github.com/webcc/cassandra-jpa
} 
- NoSQL Storage: storage for large amounts of data like sensor data.

- SQL Storage: storage for normal amounts of data like user profiles, device profiles, etc.

- Distributed File System: storage for large documents used as preprocessing results for machine learning algorithms.

\subsubsection{Cross-cutting Concerns}

Cross-cutting concerns are aspects of a software system that cannot be strictly decomposed from other components of the system.

- Access Control: handles access control to the user interface, applications, services, etc.

- Security: handles security issues like the protocol used for communicating between services or from the UI to the applications (e.g., the HTTPS protocol)

- Logging: handles logging information.

- Internationalization: handles internationalization.

- Caching: handles caching, e.g., caching of search results, data analysis, etc.

\subsection{Architecture components}

Figure 2 shows how the components of the system interact with each other.

- Sensors: different sensors involved in the system, categorized into "environment sensors" and "body sensors", e.g., heartrate sensors, blood-pressure sensors, etc.

- Platform: the central server for the system. It has the following subcomponents: User Interfaces, Services and Databases.

- Hub: a software component at the home of the patient that connects to the sensors and read their values. The connection is preferably created via Bluetooth Low Energy (BLE), although standard Bluetooth may also be used whenever it proves to be the most reliable and cost-effective alternative.

- Mobile App: a mobile application used by the patient, e.g., for providing measurements. The application communicates with the Hub (e.g., to read sensors directly) or with the Platform (e.g., to obtain historical sensor data).

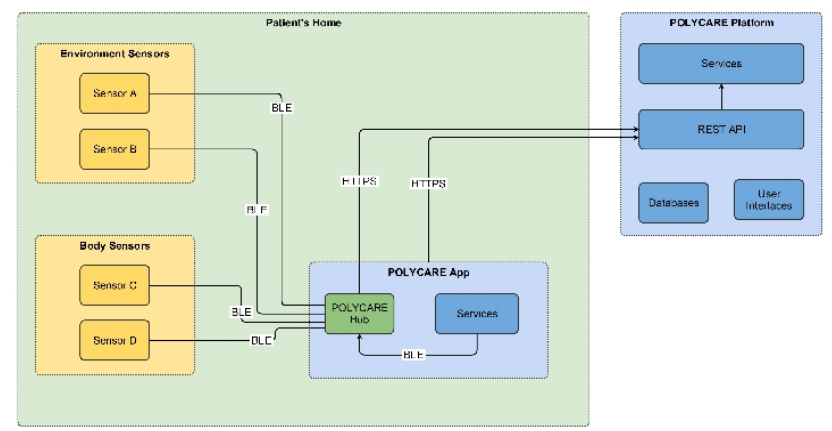

Figure 2: Relationship among the architecture components.

\section{TECHNOLOGIES}

This section will describe in detail the key core technologies that will be used for the implementation of all components and systems of the framework. We have grouped the technologies according to categories related to the different architectural components of the project. As some architectural components, this list can be extended or modified depending upon technological developments that may happen within the lifetime of the project.

\subsection{Sensors and Wearables}

The sensors will be easy-to-use for the patient and caregivers, flexible, configurable and able to measure several biomedical parameters relevant to monitor the progress of the patient.

Therefore, there is a need to use several sensors that use a wide range of technologies. The platform will include the communication with an oximeter for the monitoring of the oxygen saturation, glucometer, blood pressure sensor, electrocardiogram sensor, temperature sensor, accelerometer to measure inertial movements and respiration sensor.

The communication will be made via Bluetooth Classic or Bluetooth Low Energy (BLE). Both these technologies operate wirelessly on the ISM band from 2.4 to $2.485 \mathrm{GHz}$ band. However they have some differences, namely in the physical layer where the Bluetooth Classic has a channel spacing of $1 \mathrm{MHz}$ and hops over 79 channels while the Bluetooth Low Energy has a $2 \mathrm{MHz}$ channel spacing and only 37 channels. Among other implementation differences this make the Bluetooth Classic a technology with considerable higher throughput, which although more power "thirsty", is still more suitable for many real-time data streaming applications. On the other hand, Bluetooth Low Energy has a much lower power consumption but also a much lower data throughput.

\subsection{Decision Support System (DSS)}

In the following, we will describe the technical architecture of the DSS (see Figure 3).

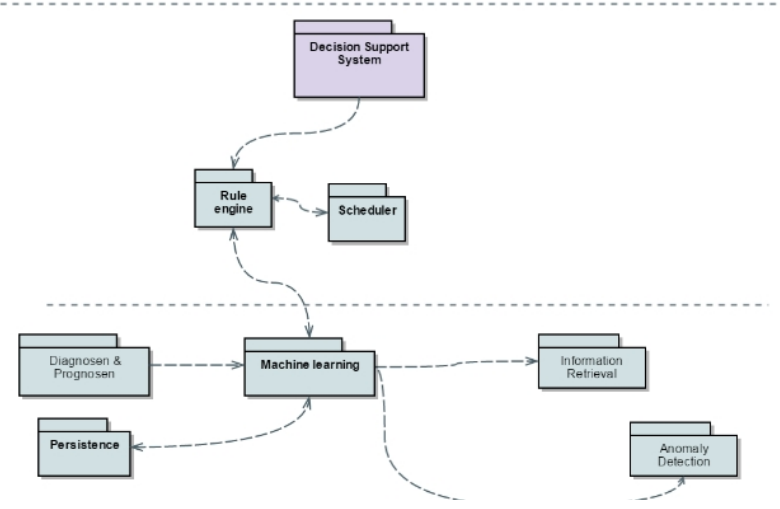

Figure 3: Overview of DSS components

As shown in Figure 3

Figure 3: Overview of DSS components the DSS will comprise of many components:

- Rule Engine: The system will require a common enterprisewide rules definition and access engine. The term "rule" is used at the highest conceptual level, as rules should be applied to different scenarios.

- Scheduler: The scheduler is the runtime watchdog of the rule engine. It monitors existing rules to verify if there condition parts is matching, then their action part would fire in a coordinated manner considering other system wide circumstances and time restrictions. 


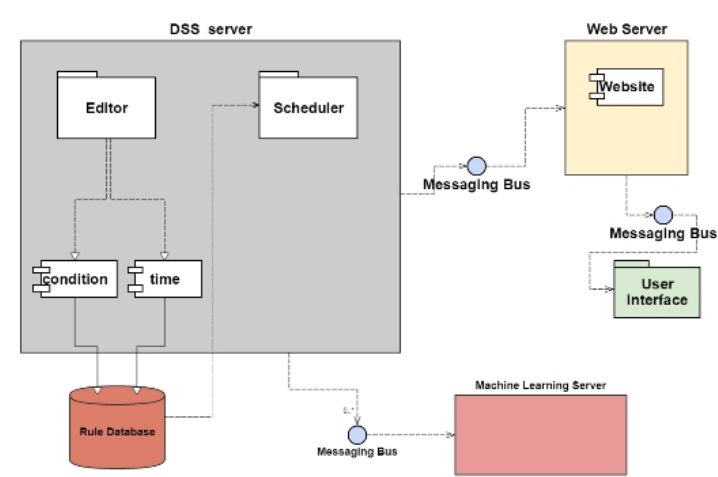

Figure 4: Deployment Diagram

As shown in Figure 4 the DSS will be deployed on the DSS server including the server side editor components for editing rules, the actions and time slots. On the other side, the DSS server will host the scheduler, which will monitor the matching rule conditions to fire them with the appropriate actions.

The rule scheduler will rely on the rules created by the rule editor. The rule engine will be implemented based on the known rule engine framework Drools ${ }^{8}$. Drools is a Business Rules Management System (BRMS) solution. It provides a core Business Rules Engine (BRE), a web authoring and rules management application.

Rules have a structure as follows (see Figure 5):

If (list of conditions) then (List of Actions)

E.g. if $($ Temp $>38 \mathrm{C}(100.4 \mathrm{~F})$ or Temp $<35.5 \mathrm{C}(96.8 \mathrm{~F}))$ then issue a body temperature alert

If $((($ heartRate $)>90)$ and (activity $<2))$ then issue a hearRate alert

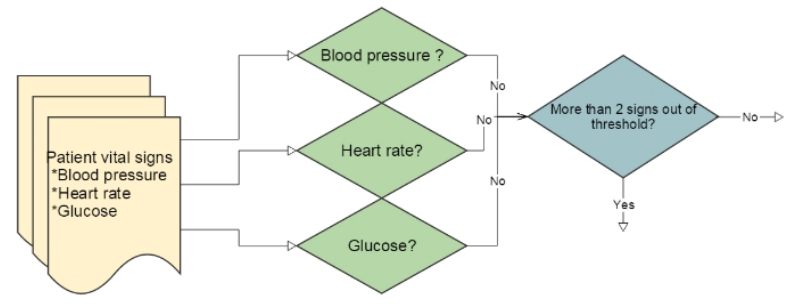

Figure 5: Overview of a possible rule

Drools rules can be expressed in either a drl file or an Excel file. Drools provides a user-friendly decision table interface. These approaches also provide visibility of the business logic to a wider audience instead of embedding the business logic in Java or custom syntax.

\subsection{Machine Learning}

Machine learning (term that is frequently confused with Big Data) is a field from computer science targeted to the autonomous recognition of patterns and trends when analysing big amounts of data. In recent years, due to the existence of ubiquitous and pervasive sensor environments for different scientific aspects, this large amount of data was too complex to be addressed by traditional data processing applications. This problem has been tackled with the creation of many libraries, frameworks, methodologies and

\footnotetext{
${ }^{8} \mathrm{http}: / /$ www.drools.org/

${ }^{9} \mathrm{http}: / /$ spark.apache.org/
}

storage technologies that facilitate the analysis of such data sets combined with known and improved machine learning algorithms.

Within the system, information of the about different health parameters will be gathered through sensors and other information sources. These data sets will be stored in our persistence layer (see section 5.9). To process these data sets, we will use Apache Spark as a processing engine. Apache Spark is an in-memory processing engine tailored for large-scale data processing, which extends the MapReduce processing model. Its main characteristics are [3]:

- A unified stack model to integrate many components.

- Access to diverse data sources such as relational databases (SQL) with structured data, NoSQL data sources (such as Apache Cassandra or Apache HBase) or even to the Hadoop Distributed Filesystem (HDFS).

- A flexible API that supports different programming languages.

- Possibility to cluster different nodes, providing a reliable and scalable system.

- Integration of standard machine learning and graph processing libraries.

- Through its streaming API, it is possible to analyse data as they are generated.

Figure 6 shows an overview of the Apache Spark components and their interrelation between them.

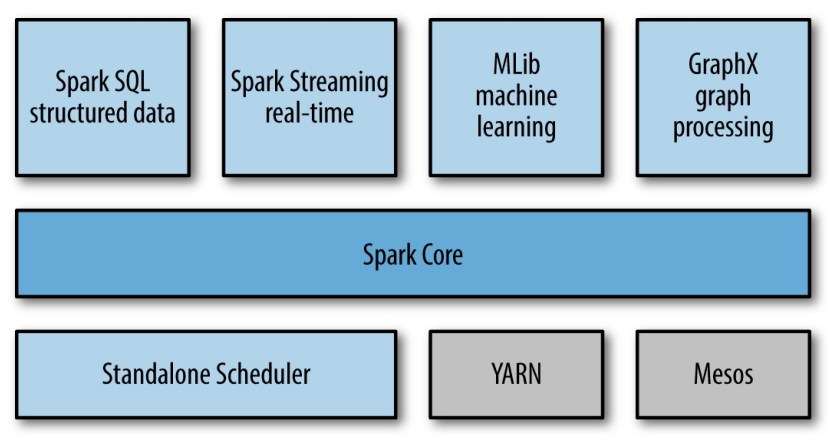

Figure 6: Spark stack of components ([3]).

The system's event processing involves tracking and analysing streams of data from events to support better insight and decisionmaking. As the data volume and diversity of data sources will be high, this goal will be challenging.

The system's event processing combines data from multiple sources to identify patterns and complex relationships across various events. The value of this processing is that it will help to identify patterns, anomalies and provides real-time alerts to act on them.

Like all problems in the analytic world, this kind of processing is also complicated by the exponential growth of data. To tackle these challenges, we intend as mentioned before utilise the open source machine learning framework Apache Spark ${ }^{9}$ and related projects. It will serve as a foundation for processing of big data via integration with an open source business-rules execution engine (Drools) used to implement the DSS and a messaging bus (RabbitMQ ${ }^{10}$ ) for interaction between these components (See Figure 7).

\footnotetext{
${ }^{10} \mathrm{https}: / /$ www.rabbitmq.com/
} 
- Ingest: RabbitMQ to ingest events

- Storage: Apache Cassandra to store and retrieve events

- Alerting: Alerts via RabbitMQ or other direct API integrations.

- Stream processing: Event processing is done with Spark Streaming. The processing occurs in micro-batches and involves parsing, lookup, persistence, building of current state from a series of historical events, and custom processing logic. It will join various streams of Spark Datasets over a slidingwindow interval and gain insights about trends in near realtime. The batching can be as frequent as every second, leading to an end-to-end latency of less than a few seconds.

- Business process management: The DSS is based as mentioned above on Drools.

- Metrics: A dashboard (collaborative Environment) based on the time-series database Apache Cassandra will provide metrics.

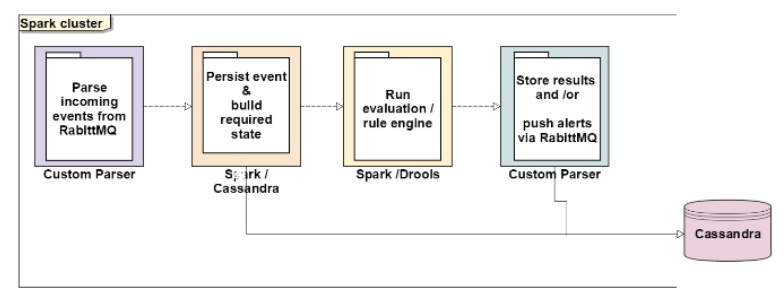

Figure 7: Polycare machine learning deployment

\subsection{Middleware}

Middleware components will be developed using the Node.js runtime. Node.js is a JavaScript runtime built on Chrome's V8 JavaScript engine. Node.js uses an event-driven, non-blocking I/O model that makes it lightweight and efficient. Node.js' package ecosystem, npm, is the largest ecosystem of open source libraries in the world. Through this runtime and a very complete package ecosystem, our development efforts will be optimised.

The hub software will consist of the Sensor middleware and the sensor service component. The persistence layer will utilise the Sensor object model for the storage and retrieval of related data. The hub software will be deployable on a computer e.g. a Raspberry PI, a tablet device etc., the hub server must support Bluetooth low energy (BLE) connectivity. The BLE connectivity is necessary for the communication with the sensor platform.

The hub server will interact with other components through a messaging bus. The following software frameworks are being used in the development of the Hub software:

- Apache Cordova: a mobile application development framework (https://cordova.apache.org/) for developing cross-platform mobile applications.

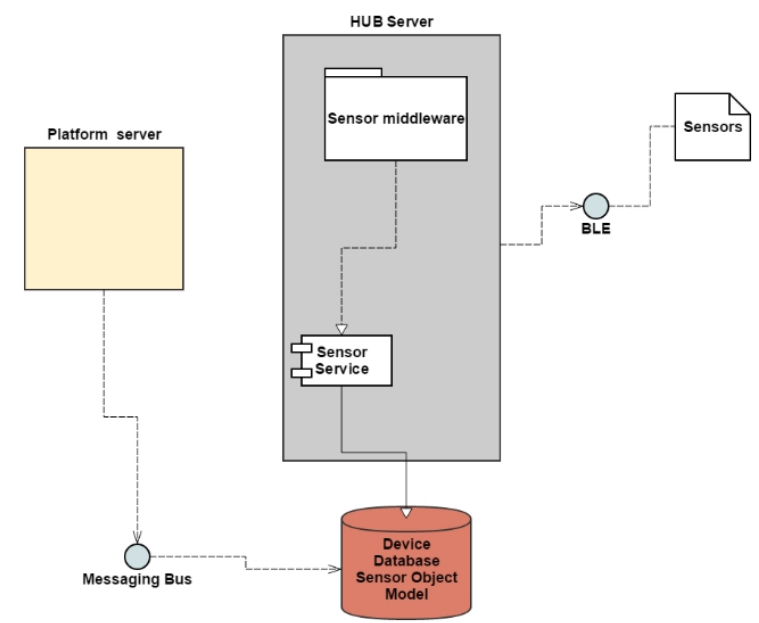

Figure 8: Deployment overview of the Hub software

- Web Bluetooth API: Custom implementation of the W3C Web Bluetooth API (https://webbluetoothcg.github.io/webbluetooth/) developed by the authors (Fraunhofer Institute FIT).

- Web Bluetooth API Plugin: plugin for Apache Cordova to connect to Bluetooth devices via BLE (Bluetooth Low Enery).

- Generic Sensor API: Custom implementation of the W3C Generic Sensor API. ${ }^{11}$

- Node.js: a JavaScript runtime environment (https://nodejs.org/en/) for executing JavaScript out of the browser (e.g., on a server or as a background process).

- noble: a Node.js module for connecting to BLE peripherals (https://github.com/sandeepmistry/noble).

- Cylon.js: a Node.js module for communicating with differents sorts of devices and sensors.

- webstomp-client: a client (https://github.com/JSteunou/webstomp-client) for the STOMP protocol to communicate with the RabbitMQ messaging system.

- amqplib: a client (https://github.com/squaremo/amqp.node) for the AMQP protocol to communicate with the RabbitMQ messaging system.

\subsection{Messaging}

For implementing the messaging component, the open source message broker software RabbitMQ (http://www.rabbitmq.com/) will be used. It implements the Advanced Message Queuing Protocol (AMQP), is written in Erlang and provides client libraries for a number of programming languages, among them Java and JavaScript, which are the two programming languages that are mainly used throughout the project.

\footnotetext{
${ }^{11} \mathrm{https}: / / w 3 \mathrm{c}$. github.io/sensors/
} 
RabbitMQ defines different components as shown in the following figure and are described in the following table: Producer/Publisher, Consumer, Exchanges, Queues, Messages, Connections, Channels, Bindings and Routing keys (See Figure 9).

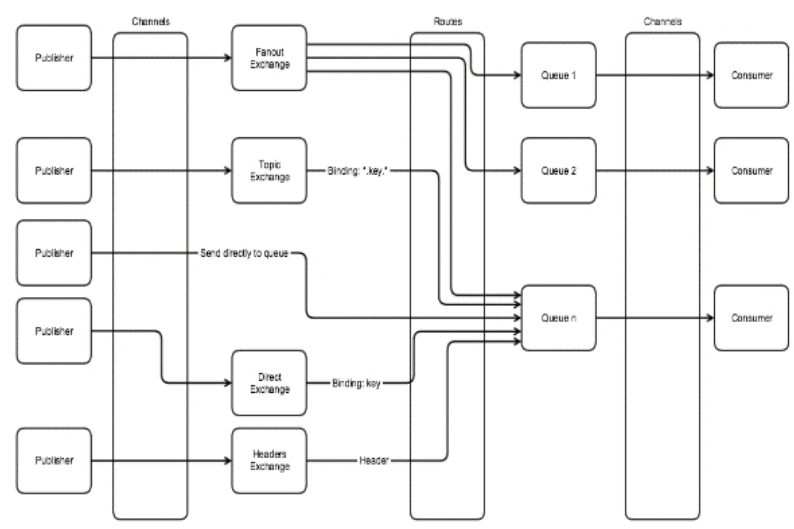

Figure 9: Messaging Topology

\subsection{Persistence}

For data persistence, will use two types of databases: SQL and NoSQL. For traditional SQL repositories, we will use reliable and established open source databases such as PostgreSQL ${ }^{12}$ or MySQL. ${ }^{13}$

For NoSQL storage, we will use Apache Cassandra. ${ }^{14}$ Cassandra has become a widely used platform for NoSQL storage due to its performance and clustering/scalability capabilities. Furthermore, it offers a flexible data model that responds to the needs of many large industrial and research projects.

\section{CONCLUSIONS AND FUTURE WORK}

This paper has presented a novel approach of a WoT eHealth architecture and an instance based on that architecture. The presented architecture was very useful as a guide for the creation of the presented instance and served as communication tool between the different stakeholders. The main lessons learnt from this exercise were that simplicity and clearness are the main features of an architecture in order to use it for communication with all stakeholders. Now we are implementing the presented instance, starting by the gathering of data in the Tele monitoring module, the development of the interpretation modules and the user interfaces, and will present a first prototype by end of 2016 .

\section{ACKNOWLEDGMENTS}

This work was partially funded by the European Commission H2020 programme; Contract number 690367; POLYCARE Polystakeholders integrated care for chronic patients in acute phases. The authors would like to acknowledge the support of the POLYCARE consortium.

\section{REFERENCES}

[1] M. Ersue; D. Romascanu; J. Schoenwaelder; A. Sehgal. "Management of Networks with Constrained Devices: Use Cases". IETF Internet Draft $<$ draft-ietf-opsawg-coman-usecases>. (4 July 2014)

[2] R.T. Fielding. Architectural Styles and the Design of Network-based Software Architectures. PhD Thesis. University of California, Irvine. http://www.ics.uci.edu/ fielding/pubs/dissertation/top.htm (2000)

[3] H. Karau, A. Konwinski, P. Wendell, M. Zaharia. Learning Spark: Lightning-Fast Big Data Analysis. O'Reilly Media. ISBN-13: 978-1449358624. (2015)

[4] T. White. Hadoop: The Definitive Guide, 4th Edition. Storage and Analysis at Internet Scale. O'Reilly Media. ISBN-13: 978-1449311520. (2015)

\footnotetext{
12 https://www.postgresql.org/

${ }^{13} \mathrm{http} / /$ www.oracle.com/us/products/mysql/overview/index.html
} 